\title{
New Results on Synchronization of Fractional-Order Memristor-Based Neural Networks via State Feedback Control
}

\author{
Xiaofan Li $\mathbb{D}^{1,2}$ Yuan Ge ${ }^{10},{ }^{1}$ Hongjian Liu, ${ }^{1}$ Huiyuan $\mathrm{Li}^{3}{ }^{3}$ and Jian-an Fang ${ }^{3}$ \\ ${ }^{1}$ Key Laboratory of Advanced Perception and Intelligent Control of High-end Equipment, Ministry of Education, \\ Anhui Polytechnic University, Wuhu 241000, China \\ ${ }^{2}$ School of Electrical Engineering, Yancheng Institute of Technology, Yancheng 224051, China \\ ${ }^{3}$ College of Information Science and Technology, Donghua University, Shanghai 201620, China \\ Correspondence should be addressed to Yuan Ge; geyuan@ahpu.edu.cn
}

Received 13 May 2020; Accepted 22 July 2020; Published 9 September 2020

Academic Editor: Xianming Zhang

Copyright (c) 2020 Xiaofan Li et al. This is an open access article distributed under the Creative Commons Attribution License, which permits unrestricted use, distribution, and reproduction in any medium, provided the original work is properly cited.

\begin{abstract}
This paper addresses the synchronization issue for the drive-response fractional-order memristor-based neural networks (FOMNNs) via state feedback control. To achieve the synchronization for considered drive-response FOMNNs, two feedback controllers are introduced. Then, by adopting nonsmooth analysis, fractional Lyapunov's direct method, Young inequality, and fractional-order differential inclusions, several algebraic sufficient criteria are obtained for guaranteeing the synchronization of the drive-response FOMNNs. Lastly, for illustrating the effectiveness of the obtained theoretical results, an example is given.
\end{abstract}

\section{Introduction}

In recent years, fractional calculus has become a useful tool in the analysis of slow relaxation phenomena. As we all know, fractional derivative has two main advantages: infinite memory and more degrees of freedom $[1,2]$. Hence, fractional derivative plays a critical part in the depiction of memory and hereditary characteristics of multifarious processes. Compared with dynamic systems described by the classical integer-order derivative, dynamic systems described by fractional derivative can accurately reflect the actual dynamic properties of real systems due to their memory and hereditary characteristics. Recently, as an extension of the classical integer-order calculus, fractional calculus has many practical applications in many interdisciplinary areas, such as fractional-order sinusoidal oscillators [3], transient wave propagation [4], fractional relaxation-oscillation and fractional diffusion-wave phenomena [5], drug release and absorption [6], and so on. Moreover, dynamic behaviors of fractional-order systems have attracted the attention of many researchers because of their practical applications. In the past decade, the dynamic analysis of the fractional-order systems has achieved many outstanding results [7-10].

In the past few years, dynamic behaviors of neural networks (NNs) have gained many attentions [11-21], since NNs have lots of applications [22, 23]. In addition, fractional derivative has been introduced to NNs, and dynamic analysis of fractional-order neural networks (FONNs) has become a focus of research and many results have been obtained $[24,25]$. Among these dynamic behaviors, as a significant dynamic characteristic, synchronization was firstly introduced [26]. Since then, research on synchronization of NNs has become a hot topic because of their wide potential applications in a large number of real systems $[27,28]$.

On the other hand, memristor was firstly predicted by Chua [29], and a practical memristor device was successfully obtained [30]. The memristor exhibits the characteristics of pinch hysteresis, which is possessed by the human brain. It is more practical to construct artificial NNs by replacing the resistor with the memristor, that is, memristor-based neural 
networks (MNNs). In recent years, dynamic behaviors of MNNs have caused widespread concern around the world [31-34]. Accordingly, it is very valuable to study the synchronization problem for fractional-order memristor-based neural networks (FOMNNs), and many excellent works have been conducted $[35,36]$.

Inspired by the discussions given above, this paper studies the synchronization issue for FOMNNs via feedback control. Firstly, to achieve the synchronization for considered drive-response FOMNNs, two feedback controllers are introduced. Then, by adopting nonsmooth analysis, fractional Lyapunov's direct method and Young inequality, and fractional-order differential inclusions, several algebraic sufficient criteria are obtained for guaranteeing the synchronization for the drive-response FOMNNs.

\section{Preliminaries and Model Description}

The following preliminaries on fractional calculus are recalled.

Definition 1 (see [37]). Given an arbitrary integrable function $\chi(t)$, its Riemann-Liouville fractional integral with fractional order $\alpha>0$ is defined as

$$
I^{\alpha} \chi(t)=\frac{1}{\Gamma(\alpha)} \int_{t_{0}}^{t}(t-s)^{\alpha-1} \chi(s) \mathrm{d} s,
$$

where $t \geq t_{0}, \Gamma(\alpha)=\int_{0}^{+\infty} s^{\alpha-1} e^{-s} \mathrm{~d} s$.

Definition 2 (see [37]). Given an arbitrary differentiable function $\chi(t)$, its Caputo fractional derivative is defined as

$$
{ }_{t_{0}}^{C} D_{t}^{\alpha} \chi(t)=\frac{1}{\Gamma(k-\alpha)} \int_{t_{0}}^{t}(t-s)^{k-\alpha-1} \chi^{(k)}(s) \mathrm{d} s,
$$

where the fractional order $\alpha>0, t \geq t_{0}, \alpha \in(k-1, k), k$ is a positive integer.

Consider an FOMNN as follows:

$$
{ }_{0}^{C} D_{t}^{\alpha} r_{l}(t)=-a_{l} r_{l}(t)+\sum_{m=1}^{n} b_{l m}\left(r_{m}(t)\right) g_{m}\left(r_{m}(t)\right)+J_{l},
$$

where $a_{l}>0$ is the self-inhibition, ${ }_{0}^{C} D_{t}^{\alpha} r_{l}(t)$ refers to the Caputo fractional derivative, $0<\alpha<1, r_{l}(t)$ refers to the state, $g_{m}\left(r_{m}(t)\right)$ refers to the activation function, $J_{l}$ is an external input or bias, and $b_{l m}\left(r_{m}(t)\right)$ is the memristor connection weight. The initial value of (3) is $r(0)=\left(r_{1}(0), r_{2}(0), \ldots, r_{n}(0)\right)^{T} \in \mathbb{R}^{n}$ and $l \in \mathbb{N}$.

The memristor connection weight $b_{l m}\left(r_{m}(t)\right)$ switches among different numbers, which can be simply modeled as follows:

$$
b_{l m}\left(r_{m}(t)\right)= \begin{cases}b_{l m}^{*}, & \left|r_{m}(t)\right| \leq \Upsilon_{l}, \\ b_{l m}^{* *}, & \left|r_{m}(t)\right|>\Upsilon_{l},\end{cases}
$$

where the switching jump $\Upsilon_{l}>0$ and $b_{l m}^{*}$ and $b_{l m}^{* *}$ are constant numbers.

$$
\begin{aligned}
& \text { Let } \underline{b}_{l m}=\min \left\{b_{l m}^{*}, b_{l m}^{* *}\right\}, \bar{b}_{l m}=\max \left\{b_{l m}^{*}, b_{l m}^{* *}\right\}, \text { and } \\
& \operatorname{co}\left[b_{l m}\left(r_{m}(t)\right)\right]= \begin{cases}b_{l m}^{*}, & \left|r_{m}(t)\right|<\Upsilon_{l}, \\
b_{l m}^{* *}, & \left|r_{m}(t)\right|>\Upsilon_{l}, \\
{\left[\underline{b}_{l m}, \bar{b}_{l m}\right],} & \left|r_{m}(t)\right|=\Upsilon_{l} .\end{cases}
\end{aligned}
$$

Then, according to the theories of differential inclusion and set-valued map, for (3), we have

$$
{ }_{0}^{C} D_{t}^{\alpha} r_{l}(t) \epsilon-a_{l} r_{l}(t)+\sum_{m=1}^{n} \mathrm{co}\left[b_{l m}\left(r_{m}(t)\right)\right] g_{m}\left(r_{m}(t)\right)+J_{l} .
$$

Or equivalently, there exists $\breve{b}_{l m}\left(r_{m}(t)\right) \in \operatorname{co}\left[b_{l m}\left(r_{m}(t)\right)\right]$, such that

$$
{ }_{0}^{C} D_{t}^{\alpha} r_{l}(t)=-a_{l} r_{l}(t)+\sum_{m=1}^{n} \breve{b}_{l m}\left(r_{m}(t)\right) g_{m}\left(r_{m}(t)\right)+J_{l} .
$$

The drive-response synchronization is considered. The corresponding response system of the drive system (3) is

$$
{ }_{0}^{C} D_{t}^{\alpha} w_{l}(t)=-a_{l} w_{l}(t)+\sum_{m=1}^{n} b_{l m}\left(w_{m}(t)\right) g_{m}\left(w_{m}(t)\right)+J_{l}+u_{l},
$$

where $u_{l}$ denotes the state feedback controller, and the initial condition $w(s)=\left(w_{1}(0), w_{2}(0), \ldots, w_{n}(0)\right)^{T}$ and $l \in \mathbb{N}$. Similarly,

$$
{ }_{0}^{C} D_{t}^{\alpha} w_{l}(t) \epsilon-a_{l} w_{l}(t)+\sum_{m=1}^{n} \operatorname{co}\left[b_{l m}\left(w_{m}(t)\right) g_{m}\left(w_{m}(t)\right)\right]+J_{l}+u_{l} .
$$

$$
\begin{gathered}
\left.\breve{b}_{l m} \text { Or } w_{m}(t)\right) \in \operatorname{co}\left[b_{l m}\left(w_{m}(t)\right)\right] \text {, such that there exists } \\
{ }_{0}^{C} D_{t}^{\alpha} w_{l}(t)=-a_{l} w_{l}(t)+\sum_{m=1}^{n} \breve{b}_{l m}\left(w_{m}(t)\right) g_{m}\left(w_{m}(t)\right)+J_{l}+u_{l} .
\end{gathered}
$$

Assumption 1. For $\forall x, y \in \mathbb{R}$, and $l \in \mathbb{N}$, the function $g_{l}(\cdot)$ : $\mathbb{R} \longrightarrow \mathbb{R}$ is monotone nondecreasing and satisfies $g_{l}(0)=0$, $\left|g_{l}().\right| \leq \widetilde{M}_{l}$, and $\left|g_{l}(x)-g_{l}(y)\right| \leq \eta_{l}|x-y|$, where $\widetilde{M}_{l}$ and $\eta_{l}$, are positive constants.

Next, let the synchronization error $e_{l}(t)=r_{l}(t)-w_{l}(t)$. For (3) and (8), the following synchronization error dynamics system can be obtained as 


$$
\begin{aligned}
{ }_{0}^{C} D_{t}^{\alpha} e_{l}(t) & =-a_{l} e_{l}(t)+\sum_{m=1}^{n}\left[\breve{b}_{l m}\left(r_{m}(t)\right) g_{m}\left(r_{m}(t)\right)-\breve{b}_{l m}\left(w_{m}(t)\right) g_{m}\left(w_{m}(t)\right)\right]-u_{l}, \\
& =-a_{l} e_{l}(t)+\sum_{m=1}^{n} \breve{b}_{l m}\left(r_{m}(t)\right) \widetilde{\zeta}_{m}\left(e_{m}(t)\right)+\sum_{m=1}^{n}\left[\breve{b}_{l m}\left(r_{m}(t)\right)-\breve{b}_{l m}\left(w_{m}(t)\right)\right] g_{m}\left(w_{m}(t)\right)-u_{l},
\end{aligned}
$$

where $\widetilde{\zeta}_{m}\left(e_{m}(t)\right)=g_{m}\left(r_{m}(t)\right)-g_{m}\left(w_{m}(t)\right)$. According to Assumption 1, we can know that $\zeta_{m}\left(e_{m}\right)$ is also monotone nondecreasing, bounded, and $\operatorname{sgn}\left(e_{m}(t)\right) \widetilde{\zeta}_{m}\left(e_{m}(t)\right)=$ $\left|\widetilde{\zeta}_{m}\left(e_{m}(t)\right)\right| \leq \eta_{m}\left|e_{m}(t)\right|$.

Definition 3 (see [37]). The drive FOMNN (3) is globally synchronized with the response FOMNN (8), if there are two positive constants $v_{1}$ and $\nu_{2}$ such that

$$
\|e(t)\|_{l} \leq\left\{\mathscr{M}\left(e\left(t_{0}\right)\right) E_{\alpha}\left(-v_{1} t^{\alpha}\right)\right\}^{v_{2}},
$$

where $t_{0}$ denotes the initial time, $\mathscr{M}(e(t))$ is locally Lipschitz on $\mathbb{R}^{n}, \mathscr{M}(0)=0$, and $\mathscr{M}(e(t))>0$.

Lemma 1 (see [38]). Let $V(t, e(t))$ be a continuous function: $\mathbb{R}^{+} \times \mathbb{R}^{n} \longrightarrow \mathbb{R}^{+}$and satisfy ${ }_{0}^{C} D_{t}^{\alpha} V(t, e(t)) \leq \varrho V(t, e(t))$, where $0<\alpha<1$ and $\varrho$ is a constant. Then, the following inequality holds:

$$
V(t, e(t)) \leq V(0, e(0)) E_{\alpha}\left(\varrho t^{\alpha}\right) .
$$

Lemma 2 (see [39]). If $z_{3}>1, z_{4}>1$ $\left(z_{3}-1\right)\left(z_{4}-1\right)=1$, the following inequality holds:

$$
z_{1} z_{2} \leq\left(\frac{(\kappa)^{z_{3}}}{z_{3}}\right)\left|z_{1}\right|^{z_{3}}+\left(\frac{1}{z_{4} \kappa^{z_{4}}}\right)\left|z_{2}\right|^{z_{4}}
$$

where $\kappa$ is an arbitrary positive number and $\left(z_{1}, z_{2}\right) \in \mathbb{R}^{2}$.

\section{Main Results}

In this section, two state feedback controllers are provided for achieving synchronization of FOMNNs.

The following two state feedback controllers are provided:

$$
\begin{aligned}
& u_{l}(t)=\lambda_{l} e_{l}(t), \\
& u_{l}(t)=\sigma_{l} e_{l}(t)+\gamma_{l} \operatorname{sign}\left(e_{l}(t)\right),
\end{aligned}
$$

where $\lambda_{l}, \sigma_{l}$ and $\gamma_{l}$ denote the control parameters, $l \in \mathbb{N}$.

Theorem 1. By holding Assumption 1, the drive FOMNN (3) is globally synchronized with the response FOMNN (8) via the controller (15), if there are two positive constants $\varsigma_{1}$ and $\varsigma_{2}$ so that

$$
\begin{gathered}
2\left(a_{l}+\lambda_{l}\right)-\sum_{m=1}^{n} \hat{b}_{l m} \eta_{m} \varsigma_{1}+\frac{\dot{b}_{m l} \eta_{l}}{\varsigma_{1}}+\left|b_{l m}^{* *}-b_{l m}^{*}\right| \eta_{m} \varsigma_{2} \\
+\frac{\left|b_{m l}^{* *}-b_{m l}^{*}\right| \eta_{l}}{\varsigma_{2}}>0, \quad l \in \mathbb{N} .
\end{gathered}
$$

Proof. Consider the Lyapunov function,

$$
V(t, e(t))=\sum_{l=1}^{n} \frac{1}{2} e_{l}^{2}(t) .
$$

Also, by calculating the Caputo fractional derivative along the trajectory of (11) with $0<\alpha<1$, we get

$$
\begin{aligned}
{ }_{0}^{C} & D_{t}^{\alpha} V(t, e(t)) \\
\leq & \sum_{l=1}^{n} e_{l}(t){ }_{0}^{C} D_{l}^{\alpha} e_{l}(t), \\
= & \sum_{l=1}^{n} e_{l}(t)\left[-a_{l} e_{l}(t)+\sum_{m=1}^{b} \breve{b}_{l m}\left(r_{m}(t)\right) \widetilde{\zeta}_{m}\left(e_{m}(t)\right)\right. \\
& \left.+\sum_{m=1}^{n}\left[\breve{b}_{l m}\left(r_{m}(t)\right)-\breve{b}_{l m}\left(w_{m}(t)\right)\right] g_{m}\left(w_{m}(t)\right)-\lambda_{l} e_{l}(t)\right], \\
\leq & \sum_{l=1}^{n}-\left(a_{l}+\lambda_{l}\right) e_{l}^{2}(t)+\sum_{l=1}^{n} \sum_{m=1}^{n} \hat{b}_{l m}\left|e_{l}(t)\right|\left|\widetilde{\zeta}_{m}\left(e_{m}(t)\right)\right| \\
& +\sum_{l=1}^{n} \sum_{m=1}^{n}\left|\breve{b}_{l m}\left(r_{m}(t)\right)-\breve{b}_{l m}\left(w_{m}(t)\right)\right|\left|g_{m}\left(w_{m}(t)\right) \| e_{l}(t)\right| .
\end{aligned}
$$

By utilizing Lemma 2, we get

$$
\begin{aligned}
& \sum_{l=1}^{n} \sum_{m=1}^{n} \dot{b}_{l m}\left|e_{l}(t)\right|\left|\widetilde{\zeta}_{m}\left(e_{m}(t)\right)\right| \\
& \leq \sum_{l=1}^{n} \sum_{m=1}^{n} \dot{b}_{l m}\left|e_{l}(t)\right| \eta_{m}\left|e_{m}(t)\right| \\
& \leq \sum_{l=1}^{n} \sum_{m=1}^{n} \dot{b}_{l m} \eta_{m}\left[\frac{1}{2}\left(e_{l}(t) \varsigma_{1}^{(1 / 2)}\right)^{2}+\frac{1}{2}\left(e_{m}(t) \varsigma_{1}^{(-1 / 2)}\right)^{2}\right], \\
& =\sum_{l=1}^{n} \sum_{m=1}^{n} \frac{\dot{b}_{l m} \eta_{m} \varsigma_{1}}{2} e_{l}^{2}(t)+\sum_{l=1}^{n} \sum_{m=1}^{n} \frac{\dot{b}_{l m} \eta_{m}}{2 \varsigma_{1}} e_{m}^{2}(t), \\
& =\sum_{l=1}^{n} \sum_{m=1}^{n}\left(\frac{\dot{b}_{l m} \eta_{m} \varsigma_{1}}{2}+\frac{\dot{b}_{m l} \eta_{l}}{2 \varsigma_{1}}\right) e_{l}^{2}(t) .
\end{aligned}
$$

Similarly, 


$$
\begin{aligned}
& \sum_{l=1}^{n} \sum_{m=1}^{n}\left|\breve{b}_{l m}\left(r_{m}(t)\right)-\breve{b}_{l m}\left(w_{m}(t)\right)\right|\left|g_{m}\left(w_{m}(t)\right)\right|\left|e_{l}(t)\right| \\
& \leq \sum_{l=1}^{n} \sum_{m=1}^{n}\left|b_{l m}^{* *}-b_{l m}^{*}\right|\left|e_{l}(t)\right| \eta_{m}\left|e_{m}(t)\right| \\
& \leq \sum_{l=1}^{n} \sum_{m=1}^{n}\left(\left(\frac{\left|b_{l m}^{* *}-b_{l m}^{*}\right| \eta_{m} \varsigma_{2}}{2}\right)+\left(\frac{\left|b_{m l}^{* *}-b_{m l}^{*}\right| \eta_{l}}{2 \varsigma_{2}}\right)\right) e_{l}^{2}(t) .
\end{aligned}
$$

Substituting (20), (21) into (19),

$$
\begin{aligned}
{ }_{0}^{C} D_{t}^{\alpha} V(t, e(t)) & \sum_{l=1}^{n}-\left(a_{l}+\lambda_{l}\right) e_{l}^{2}(t)+\sum_{l=1}^{n} \sum_{m=1}^{n}\left(\frac{\dot{b}_{l m} \eta_{m} \varsigma_{1}}{2}\right)+\left(\frac{\dot{b}_{m l} \eta_{l}}{2 \varsigma_{1}}\right) e_{1}^{2}(t) \\
& +\sum_{l=1}^{n} \sum_{m=1}^{n}\left(\frac{\left|b_{l m}^{* *}-b_{l m}^{*}\right| \eta_{m} \varsigma_{2}}{2}\right)+\left(\frac{\left|b_{m l}^{* *}-b_{m l}^{*}\right| \eta_{l}}{2 \varsigma_{2}}\right) e_{l}^{2}(t), \\
= & \sum_{l=1}^{n}\left[-a_{1}-\lambda_{l}+\sum_{m=1}^{n}\left(\frac{\dot{b}_{l m} \eta_{m} \varsigma_{1}}{2}+\frac{\dot{b}_{m l} \eta_{l}}{2 \varsigma_{1}}\right.\right. \\
& \left.\left.+\frac{\left|b_{l m}^{* *}-b_{l m}^{*}\right| \eta_{m} \varsigma_{2}}{2}+\frac{\left|b_{m l}^{* *}-b_{m l}^{*}\right| \eta_{l}}{2 \varsigma_{2}}\right)\right] e_{l}^{2}(t) \\
\leq & -\min _{1 \leq l \leq n}\left\{2\left(a_{l}+\lambda_{l}\right)-\sum_{m=1}^{n}\left(\dot{b}_{l m} \eta_{m} \varsigma_{1}+\left(\frac{\dot{b}_{m l} \eta_{l}}{\varsigma_{1}}\right)\right.\right. \\
& \left.\left.+\left|b_{l m}^{* *}-b_{l m}^{*}\right| \eta_{m} \varsigma_{2}+\frac{\left|b_{m l}^{* *}-b_{m l}^{*}\right| \eta_{l}}{\varsigma_{2}}\right)\right\} V(t, e(t)) .
\end{aligned}
$$

According to (17), we choose a constant $\varrho>0$ so that

$$
\begin{aligned}
& -\min _{1 \leq l \leq n}\left\{2\left(a_{l}+\lambda_{l}\right)-\sum_{m=1}^{n}\left(\dot{b}_{l m} \eta_{m} \varsigma_{1}+\frac{b_{m l}^{\prime} \eta_{l}}{\varsigma_{1}}\right.\right. \\
& \left.\left.+\left|b_{l m}^{* *}-b_{l m}^{*}\right| \eta_{m} \varsigma_{2}+\frac{\left|b_{m l}^{* *}-b_{m l}^{*}\right| \eta_{l}}{\varsigma_{2}}\right)\right\} \leq-\varrho<0 .
\end{aligned}
$$

From (22) and (23), we get

$$
{ }_{0}^{C} D_{t}^{\alpha} V(t, e(t)) \leq-\varrho V(t, e(t)) .
$$

By utilizing Lemma 1, we can know

$$
V(t, e(t)) \leq V(0, e(0)) E_{\alpha}\left(-\varrho t^{\alpha}\right),
$$

namely,

$$
\|e(t)\|_{2} \leq\left[\|r(s)-w(s)\|_{2} E_{\alpha}\left(-\varrho t^{\alpha}\right)\right]^{(1 / 2)} .
$$

According to (26) and Definition 3, we can obtain that the drive FOMNN (3) is globally synchronized with the response FOMNN (8) via controller (15). The proof of Theorem 1 is completed.

Theorem 2. By holding Assumption 1, the drive FOMNN (3) is globally synchronized with the response FOMNN (8) via controller (16), if there are positive constants $\rho_{l}$ so that

$$
\sum_{m=1}^{n} \rho_{l}\left|b_{l m}^{* *}-b_{l m}^{*}\right| \tilde{M}_{m}-\rho_{l} \gamma_{l} \leq 0,
$$

$$
a_{l}+\sigma_{l}-\sum_{m=1}^{n}\left(\frac{\rho_{m} \dot{b}_{m l} \eta_{l}}{\rho_{l}}\right)>0 .
$$

Proof. Firstly, we can know that, on $t \in[0,+\infty), e_{l}(t)$ is a differentiable and continuous function. Therefore, $\left(\mathrm{d}\left|e_{l}(t)\right|\right) / \mathrm{d} t$ is piecewise continuous, and $\lim _{s \longrightarrow t^{+}}\left(\left(\mathrm{d}\left|e_{l}(s)\right|\right) / \mathrm{d} s\right)$ exits for $\forall t \in \mathbb{R}^{+}$. Then, we choose the Lyapunov function:

$$
V(t, e(t))=\sum_{l=1}^{n} \rho_{l}\left|e_{l}(t)\right| .
$$

Now, by calculating the Caputo fractional derivative along the trajectory of (11) with $0<\alpha<1$, we get

$$
\begin{aligned}
&{ }_{0}^{C} D_{t}^{\alpha} V\left(t^{+}, e\left(t^{+}\right)\right) \\
& \leq \sum_{l=1}^{n} \rho_{l} \operatorname{sign}\left(e_{l}(t)\right)_{0}^{C} D_{t}^{\alpha} e_{l}(t), \\
&=\sum_{l=1}^{n} \rho_{l} \operatorname{sign}\left(e_{l}(t)\right)\left[-a_{l} e_{l}(t)+\sum_{m=1}^{n} \breve{b}_{l m}\left(r_{m}(t)\right) \widetilde{\zeta}_{m}\left(e_{m}(t)\right)\right. \\
&+\sum_{m=1}^{n}\left[\breve{b}_{l m}\left(r_{m}(t)\right)-\breve{b}_{l m}\left(w_{m}(t)\right)\right] g_{m}\left(w_{m}(t)\right) \\
&\left.-\sigma_{l} e_{l}(t)-\gamma_{l} \operatorname{sign}\left(e_{l}(t)\right)\right] \\
& \leq \sum_{l=1}^{n}\left[-\rho_{l}\left(a_{l}+\sigma_{l}\right)\left|e_{l}(t)\right|+\sum_{m=1}^{n} \rho_{l} \hat{b}_{l m}\left|\widetilde{\zeta}_{m}\left(e_{m}(t)\right)\right|\right. \\
&\left.+\sum_{m=1}^{n} \rho_{l}\left|\breve{b}_{l m}\left(r_{m}(t)\right)-\breve{b}_{l m}\left(w_{m}(t)\right)\right|\left|g_{m}\left(w_{m}(t)\right)\right|-\rho_{l} \gamma_{l}\right] \\
& \leq \sum_{l=1}^{n}\left[-\rho_{l}\left(a_{l}+\sigma_{l}\right)\left|e_{l}(t)\right|+\sum_{m=1}^{n} \rho_{l} \hat{b}_{l m} \widetilde{\eta}_{m}\left|e_{m}(t)\right|\right. \\
&\left.+\sum_{m=1}^{n} \rho_{l}\left|b_{l m}^{* *}-b_{l m}^{* *}\right| \tilde{M}_{m}-\rho_{l} \gamma_{l}\right] . \\
& {[(t)}
\end{aligned}
$$

From (27), it follows that 


$$
\begin{aligned}
& { }_{0}^{C} D_{t}^{\alpha} V\left(t^{+}, e\left(t^{+}\right)\right) \\
& \leq \sum_{l=1}^{n}\left[-\rho_{l}\left(a_{l}+\sigma_{l}\right)\left|e_{l}(t)\right|+\sum_{m=1}^{n} \rho_{l} \dot{b}_{l m} \eta_{m}\left|e_{m}(t)\right|\right] \\
& \leq \sum_{l=1}^{n}-\rho_{l}\left(a_{l}+\sigma_{l}\right)\left|e_{l}(t)\right|+\sum_{l=1}^{n} \sum_{m=1}^{n} \rho_{m} \dot{b}_{m l} \eta_{l}\left|e_{l}(t)\right| \\
& =\sum_{l=1}^{n}-\left(a_{l}+\sigma_{l}\right) \rho_{l}\left|e_{l}(t)\right|+\sum_{l=1}^{n} \sum_{m=1}^{n}\left(\frac{\rho_{m} \hat{b}_{m l} \eta_{l}}{\rho_{l}}\right) \rho_{l}\left|e_{l}(t)\right| \\
& \leq-\min _{1 \leq l \leq n}\left\{a_{l}+\sigma_{l}-\sum_{m=1}^{n}\left(\frac{\rho_{m} \dot{b}_{m l} \eta_{l}}{\rho_{l}}\right)\right\} V(t, e(t)) .
\end{aligned}
$$

According to (28), we choose a constant $\varrho>0$ so that

$$
-\min _{1 \leq l \leq n}\left\{a_{l}+\sigma_{l}-\sum_{m=1}^{n}\left(\frac{\rho_{m} \hat{b}_{m l} \eta_{l}}{\rho_{l}}\right)\right\} \leq-\varrho<0 .
$$

From (31) and (32),

$$
{ }_{0}^{C} D_{t}^{\alpha} V(t, e(t)) \leq-\varrho V(t, e(t)) .
$$

By utilizing Lemma 1, we can know

$$
V(t, e(t)) \leq V(0, e(0)) E_{\alpha}\left(-\varrho t^{\alpha}\right),
$$

that is,

$$
\sum_{l=1}^{n} \rho_{l}\left|e_{l}(t)\right| \leq \sum_{l=1}^{n} \rho_{l}\left|r_{l}(0)-w_{l}(0)\right| E_{\alpha}\left(-\varrho t^{\alpha}\right),
$$

and then,

$$
\begin{aligned}
& \sum_{l=1}^{n}\left|e_{l}(t)\right| \\
& \leq \frac{\max _{1 \leq l \leq n}\left\{\rho_{l}\right\}}{\min _{1 \leq l \leq n}\left\{\rho_{l}\right\}} \sum_{l=1}^{n}\left|r_{l}(0)-w_{l}(0)\right| E_{\alpha}\left(-\varrho t^{\alpha}\right) \\
& =\sum_{l=1}^{n} \frac{\max _{1 \leq l \leq n}\left\{\rho_{l}\right\}}{\min _{1 \leq l \leq n}\left\{\rho_{l}\right\}}\left|r_{l}(0)-w_{l}(0)\right| E_{\alpha}\left(-\varrho t^{\alpha}\right) .
\end{aligned}
$$

According to (36) and Definition 3, we can obtain that the drive FOMNN (3) is globally synchronized with the response FOMNN (8) under the state feedback controller (16). The proof of Theorem 2 is completed.

It is well known that eigenvalue of the system matrix has a tight relation with dynamics. Next, we will obtain synchronization conditions according to it.

Theorem 3. By holding Assumption 1, the drive FOMNN (3) is globally synchronized with the response FOMNN (8) via controller (15), if

$$
\begin{aligned}
a_{l} & +\lambda_{l}-\sum_{m=1}^{n}\left(\left|b_{l m}^{* *}-b_{l m}^{*}\right| \eta_{m} \varsigma_{2}+\left(\frac{\left(\left|b_{m l}^{* *}-b_{m l}^{*}\right|\right) \eta_{l}}{\varsigma_{2}}\right)\right) \\
& -\lambda_{\max }(\Xi)>0, \quad l \in \mathbb{N},
\end{aligned}
$$

where

$$
\Xi=\left[\begin{array}{cc}
0, & \left(\hat{b}_{l m} \eta_{m}\right)_{n \times n}, \\
\left(\hat{b}_{l m} \eta_{m}\right)_{n \times n}^{T}, & 0,
\end{array}\right]_{2 n \times 2 n}
$$

Proof. Firstly, according to the matrix theory, we can easily know that $-\theta$ is also the eigenvalue of $\Xi$ if $\theta$ is its eigenvalue. Therefore, the maximum eigenvalues of matrix $\Xi$ is greater than zero, that is, $\lambda_{\max }(\Xi)>0$.

Consider the Lyapunov function,

$$
V(t, e(t))=\sum_{l=1}^{n} e_{l}^{2}(t)
$$

Then, by calculating the Caputo fractional derivative along the trajectory of $(11)$ with $0<\alpha<1$, we get

$$
\begin{aligned}
& { }_{0}^{C} D_{t}^{\alpha} V(t, e(t)) \\
& \quad \leq \sum_{l=1}^{n} 2 e_{l}(t){ }_{0}^{C} D_{t}^{\alpha} e_{l}(t) \\
& \quad=\sum_{l=1}^{n} 2 e_{l}(t)\left[-a_{l} e_{l}(t)+\sum_{m=1}^{n} \breve{b}_{l m}\left(r_{m}(t)\right) \widetilde{\zeta}_{m}\left(e_{m}(t)\right)+\sum_{m=1}^{n}\left[\breve{b}_{l m}\left(r_{m}(t)\right)-\breve{b}_{l m}\left(w_{m}(t)\right)\right] g_{m}\left(w_{m}(t)\right)-\lambda_{l} e_{l}(t)\right]
\end{aligned}
$$




$$
\begin{aligned}
\leq & \sum_{l=1}^{n}-2\left(a_{l}+\lambda_{l}\right) e_{l}^{2}(t)+\sum_{l=1}^{n} \sum_{m=1}^{n} 2 \hat{b}_{l m}\left|e_{l}(t)\right| \widetilde{\zeta}_{m}\left(e_{m}(t)\right) \mid \\
& +\sum_{l=1}^{n} \sum_{m=1}^{n} 2\left|\breve{b}_{l m}\left(r_{m}(t)\right)-\breve{b}_{l m}\left(w_{m}(t)\right)\left\|g_{m}\left(w_{m}(t)\right)\right\| e_{l}(t)\right| \leq \sum_{l=1}^{n}-2\left(a_{l}+\lambda_{l}\right) e_{l}^{2}(t) \\
& +\left(|e(t)|^{T},\left|\widetilde{\zeta}^{\prime}(e(t))\right|^{T}\right)^{T} \Xi\left(|e(t)|^{T},\left|\widetilde{\zeta}^{\prime}(e(t))\right|^{T}\right)+\sum_{l=1}^{n} \sum_{m=1}^{n}\left(\left|b_{l m}^{* *}-b_{l m}^{*}\right| \eta_{m} \varsigma_{2}+\left(\frac{\left|b_{m l}^{* *}-b_{m l}^{*}\right| \eta_{l}}{\varsigma_{2}}\right) e_{l}^{2}(t)\right) \\
\leq & \sum_{l=1}^{n}\left[-2 a_{l}-2 \lambda_{l}+\sum_{m=1}^{n}\left(\left|b_{l m}^{* *}-b_{l m}^{*}\right| \eta_{m} \varsigma_{2}+\left(\frac{\left(\left|b_{m l}^{* *}-b_{m l}^{*}\right| \eta_{l}\right)}{\varsigma_{2}}\right)\right)\right] e_{l}^{2}(t)++2 \lambda_{\max }(\Xi) \sum_{l=1}^{n} e_{l}^{2}(t) \\
\leq & -2 \min _{1 \leq l \leq n}\left\{a_{l}+\lambda_{l}-\sum_{m=1}^{n}\left(\left|b_{l m}^{* *}-b_{l m}^{*}\right| \eta_{m} \varsigma_{2}+\left(\frac{\left(\left|b_{m l}^{* *}-b_{m l}^{*}\right| \eta_{l}\right)}{\varsigma_{2}}\right)\right)-\lambda_{\max }(\Xi)\right\} V(t, e(t)),
\end{aligned}
$$

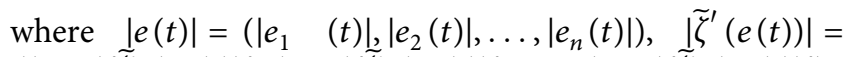
$\left(\left(1 / \eta_{1}\right)\left|\widetilde{\zeta}_{1}\left(e_{1}(t)\right)\right|,\left(1 / \eta_{2}\right)\left|\widetilde{\zeta}_{2}\left(e_{2}(t)\right)\right|, \ldots,\left(1 / \eta_{n}\right)\left|\widetilde{\zeta}_{n}\left(e_{n}(t)\right)\right|\right)$.

According to (37), we choose a constant $\varrho>0$ so that

$$
\begin{aligned}
& -\min _{1 \leq l \leq n}\left\{a_{l}+\lambda_{l}-\sum_{m=1}^{n}\left(\left|b_{l m}^{* *}-b_{l m}^{*}\right| \eta_{m} \varsigma_{2}+\left(\frac{\left|b_{m l}^{* *}-b_{m l}^{*}\right| \eta_{l}}{\varsigma_{2}}\right)\right)\right. \\
& \left.-\lambda_{\max }(\Xi)\right\} \leq-\varrho<0 .
\end{aligned}
$$

From (40) and (41), we can get

$$
{ }_{0}^{C} D_{t}^{\alpha} V(t, e(t)) \leq-2 \varrho V(t, e(t)) .
$$

By utilizing Lemma 1, we can know

$$
V(t, e(t)) \leq V(0, e(0)) E_{\alpha}\left(-2 \varrho t^{\alpha}\right),
$$

namely,

$$
\sum_{l=1}^{n} e^{2}(t) \leq \sum_{l=1}^{n}\left(r_{1}(0)-w_{1}(0)\right)^{2} E_{\alpha}\left(-2 \varrho t^{\alpha}\right),
$$

and then,

$$
\begin{aligned}
& \left(\sum_{l=1}^{n}\left(\frac{e^{2}(t)}{2}\right)\right)^{(1 / 2)} \\
& \quad \leq\left(\sum_{l=1}^{n}\left(\frac{\left(r_{l}(0)-w_{l}(0)\right)^{2}}{2} E_{\alpha}\left(-2 \varrho t^{\alpha}\right)\right)\right)^{(1 / 2)} .
\end{aligned}
$$

According to (45) and Definition 3, we can obtain that the drive FOMNN (3) is globally synchronized with the response FOMNN (8) under the state feedback controller (15). The proof of Theorem 3 is completed.

Remark 1. Free-weighting parameters $\varsigma_{1}$ and $\varsigma_{2}$ are introduced in synchronization criterion (17) of Theorem 1. Also, free-weighting parameters $\varsigma_{1}$ and $\varsigma_{2}$ can be used to reduce the conservativeness of the synchronization criterion.

Remark 2. The synchronization criteria obtained in this paper only depend on their system parameters, which are simpler in form than linear matrix inequalities [40]. In addition, these algebraic synchronization criteria are easy to check, quick to calculate, and help greatly reduce the computational burden.

\section{Numerical Simulation}

Now, we provide a numerical simulation to illustrate the effectiveness of results.

We consider the FOMNN (8) as the response system and the drive FOMNN as follows:

$$
\left\{\begin{array}{l}
{ }_{0}^{C} D_{t}^{\alpha} r_{1}(t)=-r_{1}(t)+b_{11}\left(r_{1}(t)\right) g_{1}\left(r_{1}(t)\right)+b_{12}\left(r_{2}(t)\right) g_{2}\left(r_{2}(t)\right)+J_{1}, \\
{ }_{0}^{C} D_{t}^{\alpha} r_{1}(t)=-1.2 r_{2}(t)+b_{21}\left(r_{1}(t)\right) g_{1}\left(r_{1}(t)\right)+b_{22}\left(r_{2}(t)\right) g_{2}\left(r_{2}(t)\right)+J_{2},
\end{array}\right.
$$



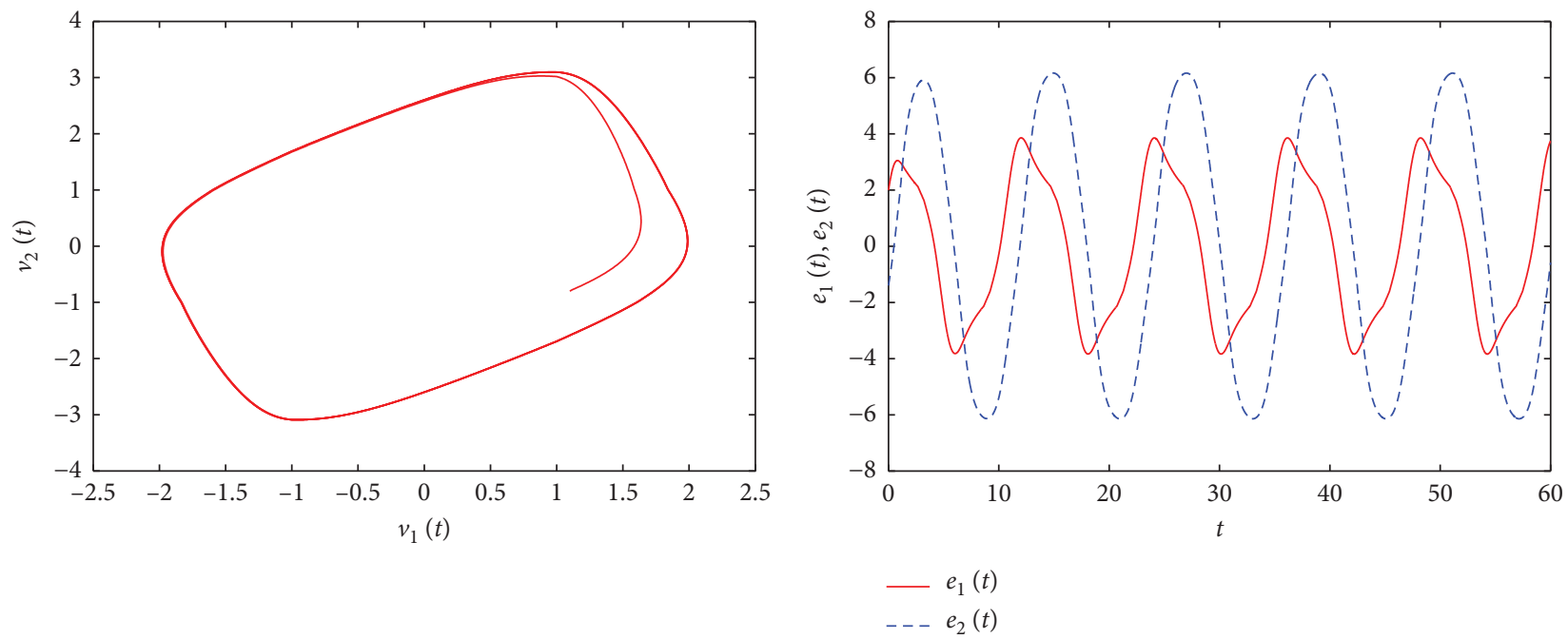

(a)
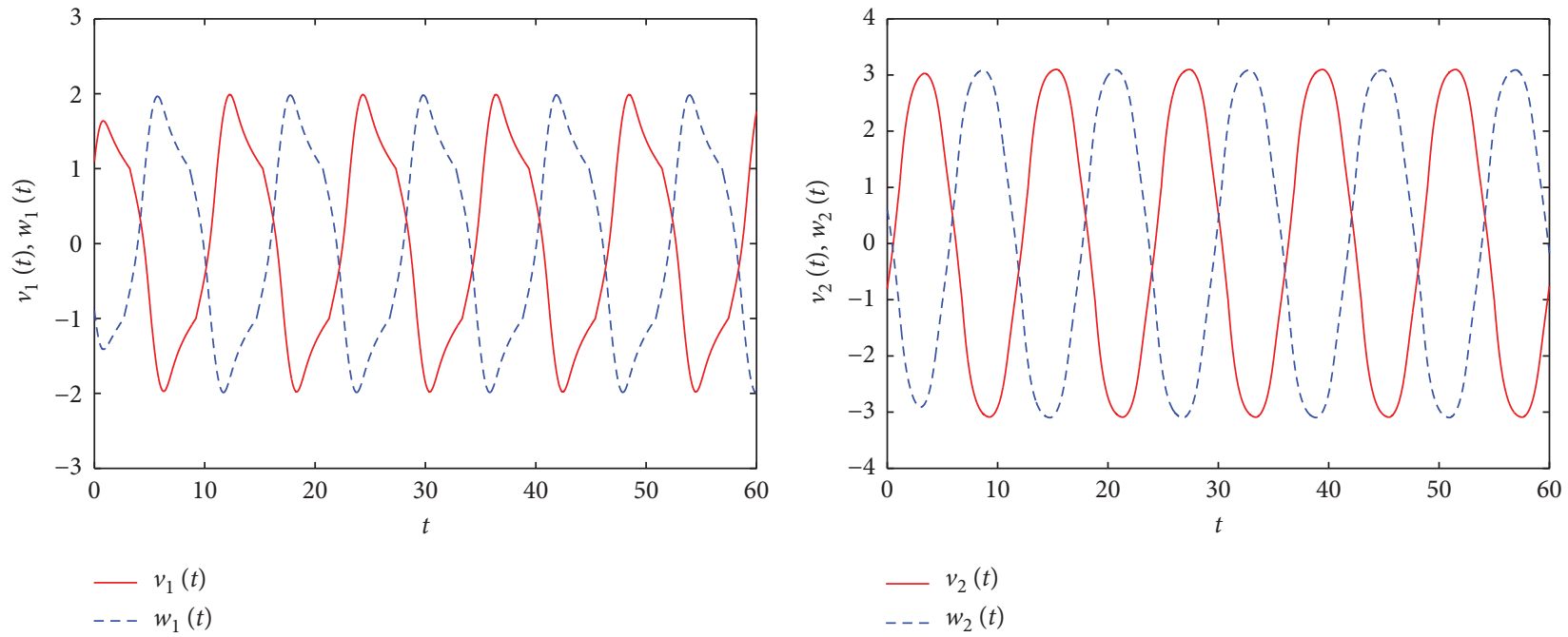

(c)

(d)

FIgURE 1: The chaotic behavior, error signal and state trajectories of the derive FOMNN (46), and the response FOMNN (8) without a controller.

where $\quad t \in[0,+\infty), J_{1}=J_{2}=0, \quad \alpha=0.98, g_{l}\left(r_{l}(t)\right)=$ $\tanh \left(r_{l}(t)\right), l=1,2$, and

$$
\begin{aligned}
& b_{11}\left(r_{1}(t)\right)= \begin{cases}2, & \left|r_{1}(t)\right| \leq 1, \\
2.2, & \left|r_{1}(t)\right|>1,\end{cases} \\
& b_{12}\left(r_{2}(t)\right)= \begin{cases}-1.2, & \left|r_{2}(t)\right| \leq 1, \\
-0.9, & \left|r_{2}(t)\right|>1,\end{cases} \\
& b_{21}\left(r_{1}(t)\right)= \begin{cases}1.8, & \left|r_{1}(t)\right| \leq 1, \\
2.1, & \left|r_{1}(t)\right|>1,\end{cases} \\
& b_{22}\left(r_{2}(t)\right)= \begin{cases}1.7, & \left|r_{2}(t)\right| \leq 1, \\
2.2, & \left|r_{2}(t)\right|>1 .\end{cases}
\end{aligned}
$$

Then, we can obtain $\dot{b}_{11}=2.2, b_{12}=1.2, b_{21}=$ $2.1, \hat{b}_{22}=2.2, \eta_{1}=\eta_{2}=1$, and $\tilde{M}_{1}=\tilde{M}_{2}=1$. Let $r_{1}(0)=$ $1.1, r_{2}(0)=-0.8, w_{1}(0)=-0.9, w_{2}(0)=0.6 ; \quad$ Figure $\quad 1$ depicts the chaotic behavior, error and state trajectories of the derive FOMNN (46), and the response FOMNN (8) without an external controller, which are not synchronous.

Now, according to (17) in Theorem 1, we can get

$$
\begin{aligned}
& 2\left(1+\lambda_{1}\right)-3.4 \varsigma_{1}-\left(\frac{4.3}{\varsigma_{1}}\right)-0.5 \varsigma_{2}-\left(\frac{0.5}{\varsigma_{2}}\right)>0, \\
& 2\left(1.2+\lambda_{2}\right)-4.3 \varsigma_{1}-\left(\frac{3.4}{\varsigma_{1}}\right)-0.8 \varsigma_{2}-\left(\frac{0.8}{\varsigma_{2}}\right)>0 .
\end{aligned}
$$

Next, we choose $\varsigma_{1}=\varsigma_{2}=1$ for convenient calculation, then we can obtain $\lambda_{1}>3.35, \lambda_{2}>3.45$, and choose $\lambda_{1}=3.4, \lambda_{2}=3.5$. The condition of Theorem 1 is satisfied, that is, the drive FOMNN (46) and the response FOMNN (8) are globally synchronized via the controller $u_{1}(t)=3.4 e_{1}(t), u_{2}(t)=3.5 e_{2}(t)$. Let $r_{1}(0)=1.1, r_{2}(0)=$ 


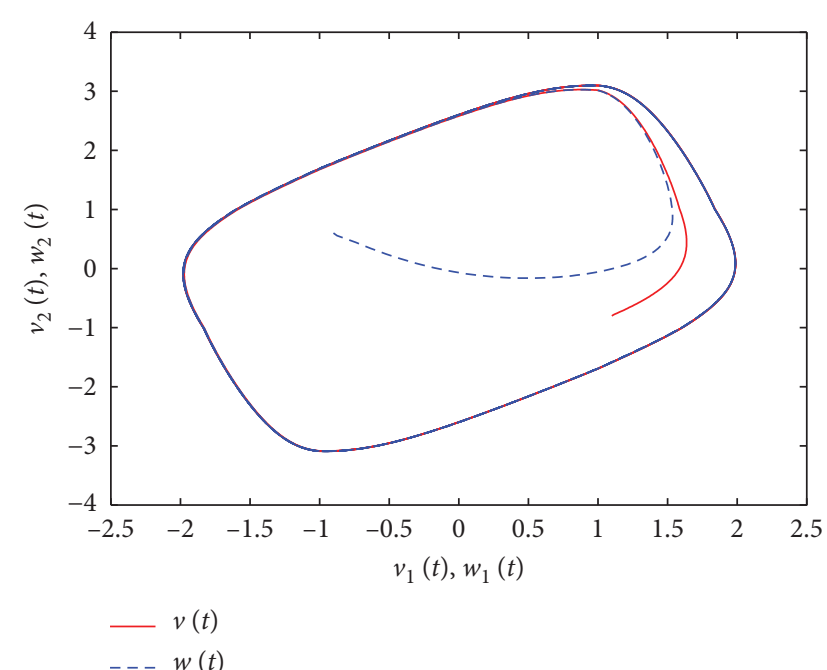

(a)

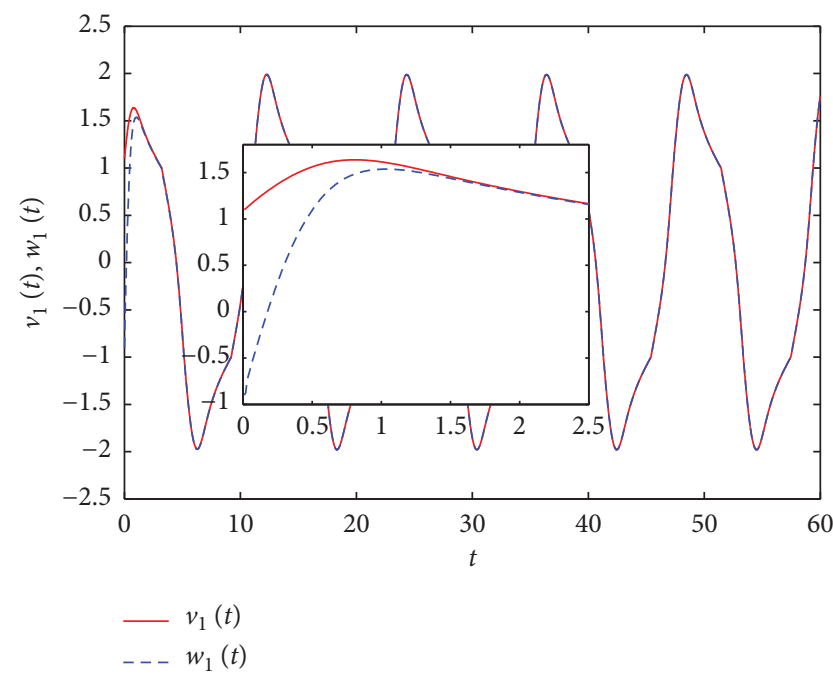

(c)

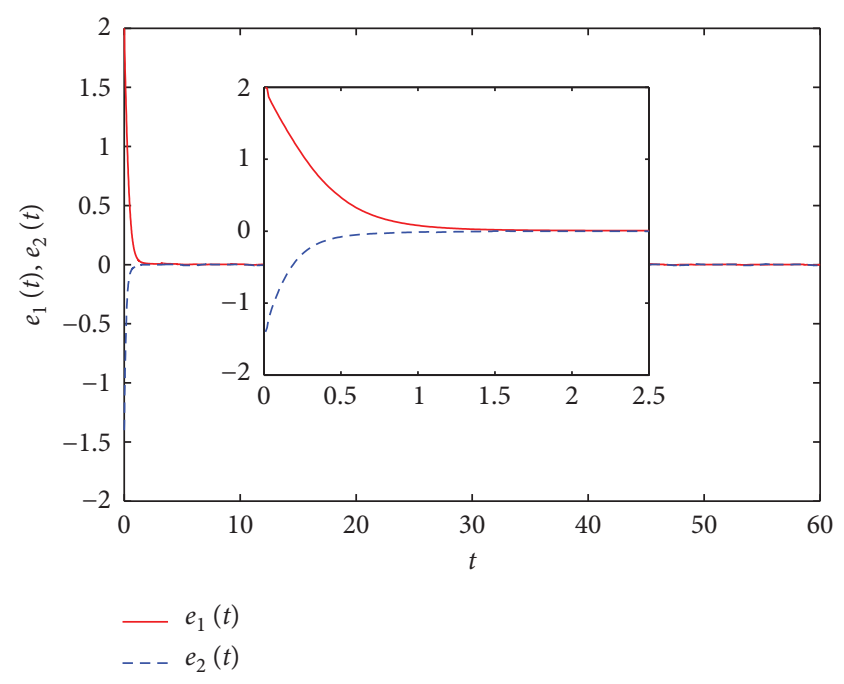

(b)

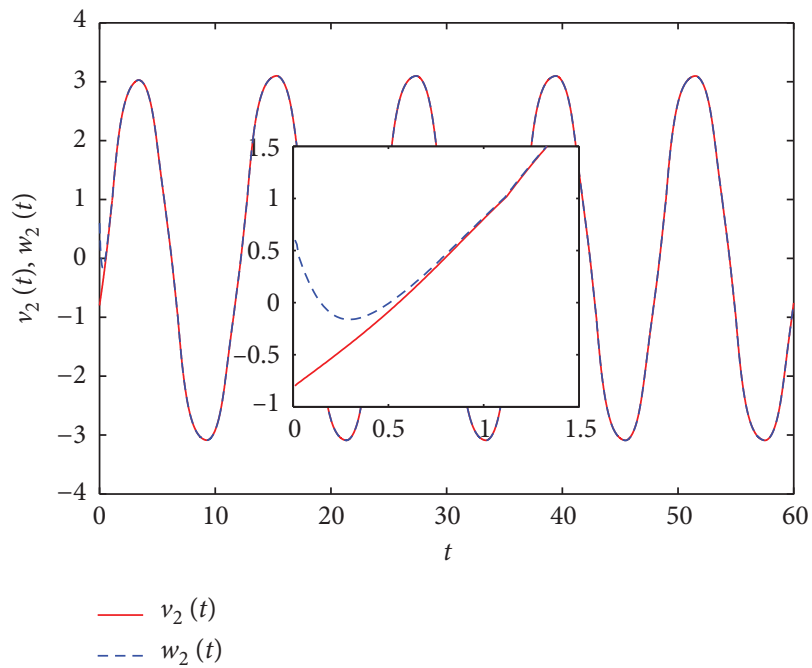

(d)

Figure 2: The chaotic behaviors, error and state trajectories of the derive FOMNN (46), and the response FOMNN (8) with the state feedback controller $u_{1}(t)=3.4 e_{1}(t), u_{2}(t)=3.5 e_{2}(t)$.

$-0.8, w_{1}(0)=-0.9, w_{2}(0)=0.6$, and then, the chaotic behaviors, error and state trajectories of the drive FOMNN (46), and the response FOMNN (8) via the controller $u_{1}(t)=$ $3.4 e_{1}(t), u_{2}(t)=3.5 e_{2}(t)$ are shown in Figure 2 .

Similarly, according to (27) in Theorem 2 and by selecting $\rho_{1}=\rho_{2}=1$, we can get $\gamma_{1} \geq 0.5, \gamma_{2} \geq 0.7$ and choose $\gamma_{1}=0.5, \gamma_{2}=0.7$. Moreover, according to (28) in Theorem 2 , we can get $\sigma_{1} \geq 3.3, \sigma_{2} \geq 2.2$ and choose $\sigma_{1}=3.4, \sigma_{2}=2.3$. The conditions of Theorem 2 are satisfied, that is, the drive FOMNN (46) and the response FOMNN (8) are globally synchronized via the controller $u_{1}(t)=3.4 e_{1}(t)+$ $0.5 \operatorname{sign}\left(e_{1}(t)\right) \quad, u_{2}(t)=2.3 e_{2}(t)+0.7 \operatorname{sign}\left(e_{1}(t)\right)$. Let $r_{1}(0)=-1.9, r_{2}(0)=-1.8, w_{1}(0)=1.2, w_{2}(0)=-0.6$; then, the chaotic behaviors, error and state trajectories of the drive FOMNN (46), and the response FOMNN (8) via the controller $\quad u_{1}(t)=3.4 e_{1}(t)+0.5 \operatorname{sign}\left(e_{1}(t)\right), u_{2}(t)=$ $2.3 e_{2}(t)+0.7 \operatorname{sign}\left(e_{2}(t)\right)$ are shown in Figure 3 .
Moreover, in Theorem 3, system parameters matrix $\Xi$ can be easily obtained as

$$
\Xi=\left[\begin{array}{cccc}
0 & 0 & 2.2 & 1.2 \\
0 & 0 & 2.1 & 2.2 \\
2.2 & 1.2 & 0 & 0 \\
2.1 & 2.2 & 0 & 0
\end{array}\right] .
$$

By easily calculating, the maximal eigenvalue of system parameters matrix $\Xi$ is $\lambda_{\max }(\Xi)=3.7875$. Next, according to (37) in Theorem 3, choosing $\varsigma_{1}=\varsigma_{2}=1$, we can obtain $\lambda_{1}>3.7875$ and $\lambda_{2}>3.9875$ and choose $\lambda_{1}=3.8, \lambda_{2}=4$. The condition of Theorem 3 is satisfied, that is, the drive FOMNN (46) and the response FOMNN (8) are globally synchronized via the controller $u_{1}(t)=3.8 e_{1}(t)$, $u_{2}(t)=4 e_{2}(t)$. Let $r_{1}(0)=1.5, r_{2}(0)=-3, w_{1}(0)=-1.3$, $w_{2}(0)=2.6$; then, the chaotic behaviors, error and state 

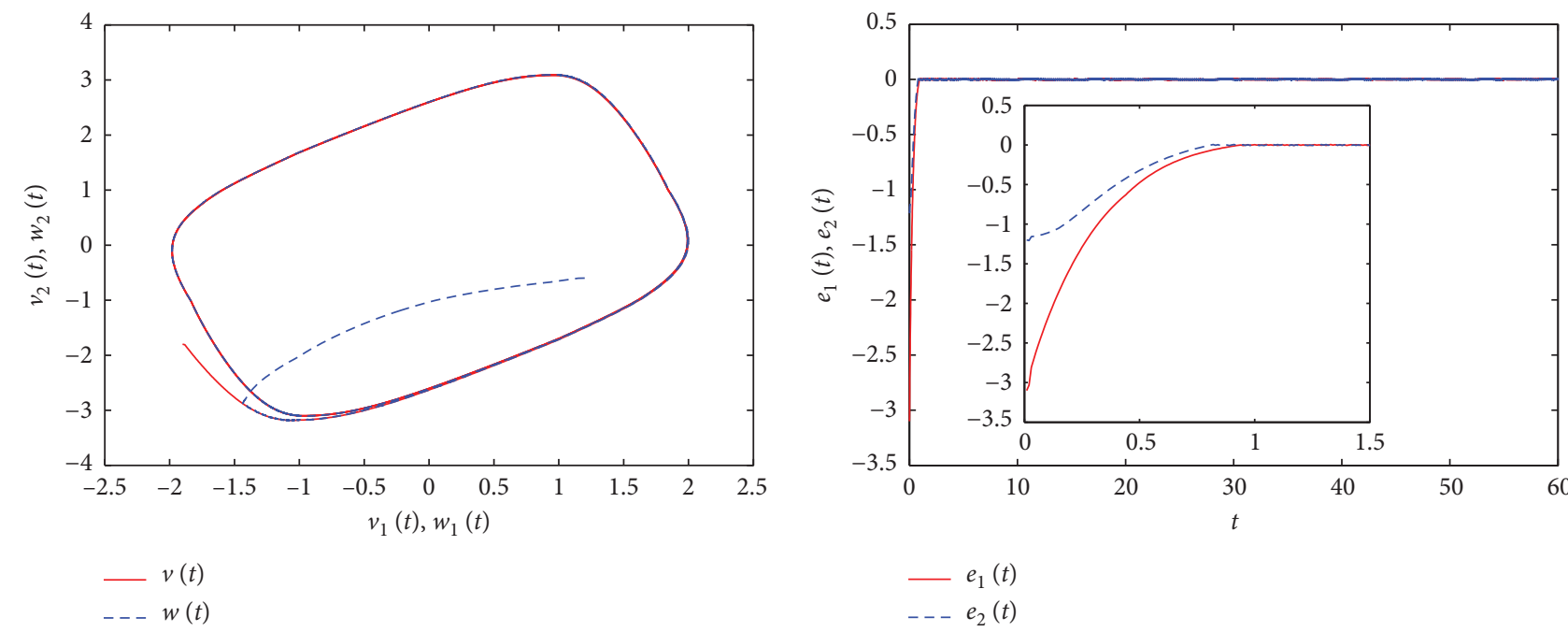

(a)

(b)
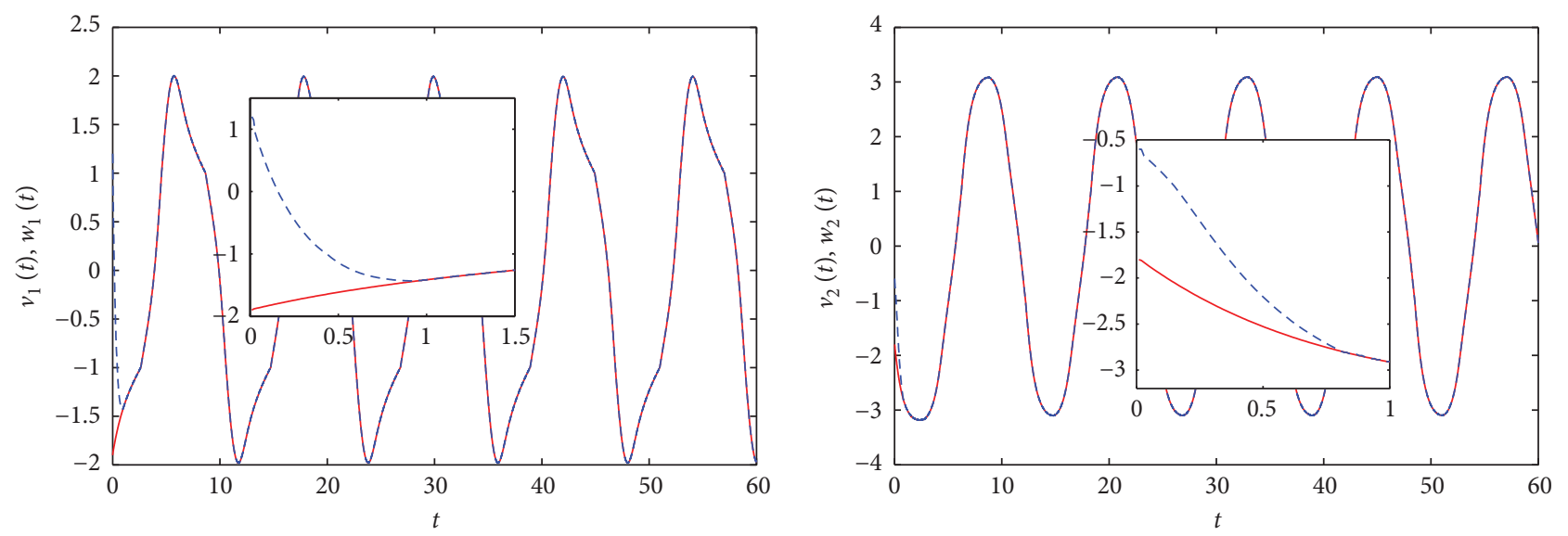

- $v_{1}(t)$

$-v_{2}(t)$

- - $w_{1}(t)$

- - $w_{2}(t)$

(c)

(d)

FIGURE 3: The chaotic behaviors, error and state trajectories of the derive FOMNN (46), and the response FOMNN (8) with the state feedback controller $u_{1}(t)=3.4 e_{1}(t)+0.5 \operatorname{sign}\left(e_{1}(t)\right), u_{2}(t)=2.3 e_{2}(t)+0.7 \operatorname{sign}\left(e_{2}(t)\right)$.
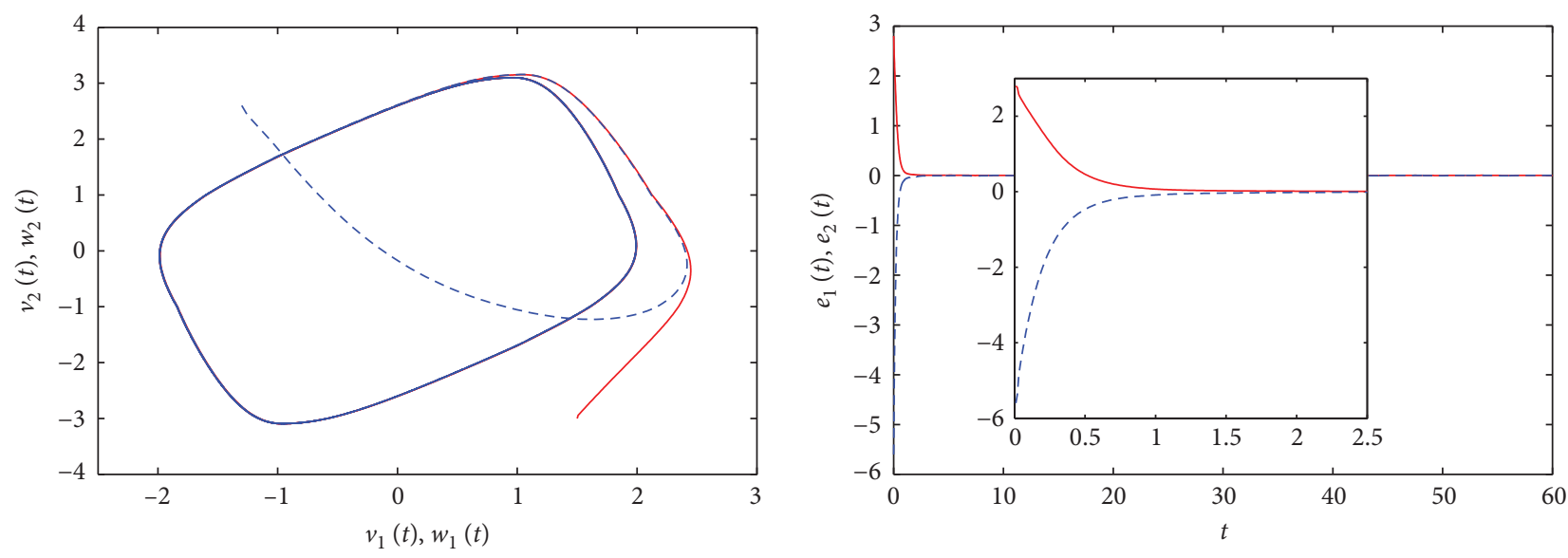

$$
\begin{aligned}
& v(t) \\
& ---w(t)
\end{aligned}
$$$$
-e_{1}(t)
$$$$
--e_{2}(t)
$$

(a)

Figure 4: Continued. 


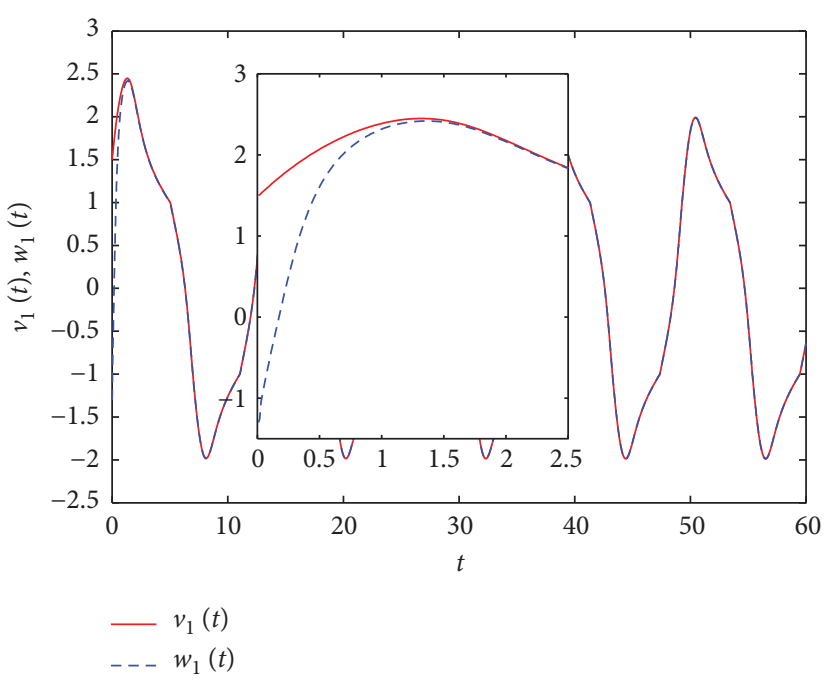

(c)

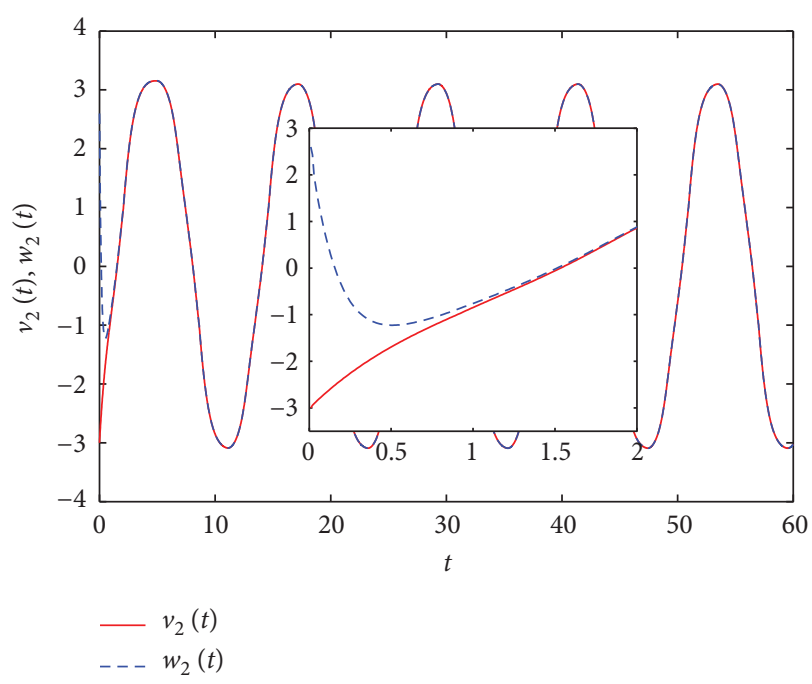

(d)

FIgURE 4: The chaotic behaviors, error and state trajectories of the derive FOMNN (46), and the response FOMNN (8) with the state feedback controller $u_{1}(t)=3.8 e_{1}(t), u_{2}(t)=4 e_{2}(t)$.

trajectories of the drive FOMNN (46), and the response FOMNN (8) via the controller $u_{1}(t)=3.8 e_{1}(t), u_{2}(t)=$ $4 e_{2}(t)$ are shown in Figure 4.

\section{Conclusions}

In this paper, the synchronization issue for FOMNNs has been investigated via state feedback control. To achieve the synchronization for the considered drive-response FOMNNs, feedback controllers are first introduced. Then, by adopting nonsmooth analysis, fractional Lyapunov's direct method and Young inequality, and fractional-order differential inclusions, several algebraic sufficient criteria are obtained for guaranteeing the synchronization for the driveresponse FOMNNs. Finally, an example is given to illustrate the effectiveness of the theoretical results. In future research, theoretical results here will be used to address the state estimation of FOMNNs.

\section{Data Availability}

The data used to support the findings of this study are included within the article.

\section{Conflicts of Interest}

The authors declare that they have no conflicts of interest.

\section{Acknowledgments}

This work was supported by the Open Research Fund of the Key Laboratory of Advanced Perception and Intelligent Control of High-end Equipment, Ministry of Education, Anhui Polytechnic University, under Grant GDSC202012.

\section{References}

[1] H. Wang, Y. Yu, and G. Wen, "Stability analysis of fractionalorder Hopfield neural networks with time delays," Neural Networks, vol. 55, pp. 98-109, 2014.

[2] B. Meng, X. Wang, Z. Zhang, and Z. Wang, "Necessary and sufficient conditions for normalization and sliding mode control of singular fractional-order systems with uncertainties," Science China Information Sciences, vol. 63, no. 5, Article ID 152202, 2020.

[3] A. G. Radwan, A. S. Elwakil, and A. M. Soliman, "Fractional-order sinusoidal oscillators: design procedure and practical examples," IEEE Transactions on Circuits and Systems I: Regular Papers, vol. 55, no. 7, pp. 2051-2063, 2008.

[4] M. Fellah, Z. E. A. Fellah, and C. Depollier, "Transient wave propagation in inhomogeneous porous materials: application of fractional derivatives," Signal Processing, vol. 86, no. 10, pp. 2658-2667, 2006.

[5] F. Mainardi, "Fractional relaxation-oscillation and fractional diffusion-wave phenomena," Chaos, Solitons \& Fractals, vol. 7, no. 9, pp. 1461-1477, 1996.

[6] J. Kytariolos, A. Dokoumetzidis, and P. Macheras, "Power law IVIVC: an application of fractional kinetics for drug release and absorption," European Journal of Pharmaceutical Sciences, vol. 41, no. 2, pp. 299-304, 2010.

[7] K. Rajagopal, L. Guessas, A. Karthikeyan, A. Srinivasan, and G. Adam, "Fractional-order and memristive nonlinear systems: advances and applications," Complexity, vol. 2017, Article ID 1892618, 19 pages, 2017.

[8] Y. Yu, H. Bao, M. Shi, B. Bao, Y. Chen, and M. Chen, "Complex dynamical behaviors of a fractional-order system based on a locally active memristor," Complexity, vol. 2019, Article ID 2051053, 13 pages, 2019.

[9] X. Li and T. Huang, "Adaptive synchronization for fuzzy inertial complex-valued neural networks with state-dependent coefficients and mixed delays," Fuzzy Sets and Systems, 2020. 
[10] W. Zhang, J. Cao, D. Chen, and A. Alsaedi, "Out lag synchronization of fractional order delayed complex networks with coupling delay via pinning control," Complexity, vol. 2019, Article ID 5612150, 7 pages, 2019.

[11] H. Li, J.-a. Fang, X. Li, and T. Huang, "Exponential synchronization of multiple impulsive discrete-time memristorbased neural networks with stochastic perturbations and time-varying delays," Neurocomputing, vol. 392, pp. 86-97, 2020.

[12] X. Li, J.-a. Fang, H. Li, and W. Duan, "Exponential stabilization of time-varying delayed complex-valued memristorbased neural networks via impulsive control," Asian Journal of Control, vol. 21, no. 6, pp. 2290-2301, 2019.

[13] W. Zhang, Y. Tang, W. K. Wong, and Q. Miao, "Stochastic stability of delayed neural networks with local impulsive effects," IEEE Transactions on Neural Networks and Learning Systems, vol. 26, no. 10, pp. 2336-2345, 2015.

[14] X. Li, W. Zhang, J.-A. Fang, and H. Li, "Event-triggered exponential synchronization for complex-valued memristive neural networks with time-varying delays," IEEE Transactions on Neural Networks and Learning Systems, 2020.

[15] Y. Fan, X. Huang, H. Shen, and J. Cao, "Switching eventtriggered control for global stabilization of delayed memristive neural networks: an exponential attenuation scheme," Neural Networks, vol. 117, pp. 216-224, 2019.

[16] J. Jia, X. Huang, Y. Li, J. Cao, and A. Alsaedi, "Global stabilization of fractional-order memristor-based neural networks with time delay," IEEE Transactions on Neural Networks and Learning Systems, vol. 31, no. 3, pp. 997-1009, 2019.

[17] Y. Fan, X. Huang, Y. Li, J. Xia, and G. Chen, "Aperiodically intermittent control for quasi-synchronization of delayed memristive neural networks: an interval matrix and matrix measure combined method," IEEE Transactions on Systems, Man, and Cybernetics: Systems, vol. 49, no. 11, pp. 2254-2265, 2018.

[18] X. Li, T. Huang, and J.-a. Fang, "Event-triggered stabilization for takagi-sugeno fuzzy complex-valued memristive neural networks with mixed time-varying delays," IEEE Transactions on Fuzzy Systems, 2020.

[19] H.-H. Lian, S.-P. Xiao, H. Yan, F. Yang, and H.-B. Zeng, "Dissipativity analysis for neural networks with time-varying delays via a delay-product-type lyapunov functional approach," IEEE Transactions on Neural Networks and Learning Systems, 2020.

[20] X.-M. Zhang, Q.-L. Han, X. Ge, and D. Ding, "An overview of recent developments in lyapunov-krasovskii functionals and stability criteria for recurrent neural networks with timevarying delays," Neurocomputing, vol. 313, pp. 392-401, 2018.

[21] X.-M. Zhang, Q.-L. Han, Z. Wang, and B.-L. Zhang, "Neuronal state estimation for neural networks with two additive time-varying delay components," IEEE Transactions on Cybernetics, vol. 47, no. 10, pp. 3184-3194, 2017.

[22] M. Egmont-Petersen, D. de Ridder, and H. Handels, "Image processing with neural networks-a review," Pattern Recognition, vol. 35, no. 10, pp. 2279-2301, 2002.

[23] Y. V. Pershin and M. Di Ventra, "Experimental demonstration of associative memory with memristive neural networks," Neural Networks, vol. 23, no. 7, pp. 881-886, 2010.

[24] Y. Tang, Z. Wang, and J. A. Fang, "Pinning control of fractional-order weighted complex networks," Chaos, vol. 19, no. 1, 2009.

[25] C. Song and J. Cao, "Dynamics in fractional-order neural networks," Neurocomputing, vol. 142, pp. 494-498, 2014.
[26] L. M. Pecora and T. L. Carroll, "Synchronization in chaotic systems," Physical Review Letters, vol. 64, no. 8, pp. 821-824, 1990.

[27] S. Wen, Z. Zeng, T. Huang, and Y. Zhang, "Exponential adaptive lag synchronization of memristive neural networks via fuzzy method and applications in pseudorandom number generators," IEEE Transactions on Fuzzy Systems, vol. 22, no. 6, pp. 1704-1713, 2014.

[28] X. Li, J.-a. Fang, and H. Li, "Exponential stabilisation of stochastic memristive neural networks under intermittent adaptive control," IET Control Theory \& Applications, vol. 11, no. 15, pp. 2432-2439, 2017.

[29] L. Chua, "Memristor-The missing circuit element," IEEE Transactions on Circuit Theory, vol. 18, no. 5, pp. 507-519, 1971.

[30] D. B. Strukov, G. S. Snider, D. R. Stewart, and R. S. Williams, "The missing memristor found," Nature, vol. 453, no. 7191, pp. 80-83, 2008.

[31] X. Li, J.-a. Fang, and H. Li, "Exponential stabilisation of memristive neural networks under intermittent output feedback control," International Journal of Control, vol. 91, no. 8, pp. 1848-1860, 2018.

[32] X. Li, J.-a. Fang, and H. Li, "Master-slave exponential synchronization of delayed complex-valued memristor-based neural networks via impulsive control," Neural Networks, vol. 93, pp. 165-175, 2017.

[33] X. Yang, Y. Feng, K. F. C. Yiu, Q. Song, and F. E. Alsaadi, "Synchronization of coupled neural networks with infinitetime distributed delays via quantized intermittent pinning control," Nonlinear Dynamics, vol. 94, no. 3, pp. 2289-2303, 2018.

[34] X. Li, J.-a. Fang, and H. Li, "Exponential synchronization of stochastic memristive recurrent neural networks under alternate state feedback control," International Journal of Control, Automation and Systems, vol. 16, no. 6, pp. 28592869, 2018.

[35] S. Yang, C. Li, and T. Huang, "Exponential stabilization and synchronization for fuzzy model of memristive neural networks by periodically intermittent control," Neural Networks, vol. 75, pp. 162-172, 2016.

[36] X. Li, J.-a. Fang, W. Zhang, and H. Li, "Finite-time synchronization of fractional-order memristive recurrent neural networks with discontinuous activation functions," Neurocomputing, vol. 316, pp. 284-293, 2018.

[37] I. Podlubny, Fractional Differential Equations, Academic Press, New York, NY, USA, 1999.

[38] J. Chen, Z. Zeng, and P. Jiang, "Global Mittag-Leffler stability and synchronization of memristor-based fractional-order neural networks," Neural Networks, vol. 51, pp. 1-8, 2014.

[39] H. Deng and M. Krstić, "Stochastic nonlinear stabilization-I: a backstepping design," Systems \& Control Letters, vol. 32, no. 3, pp. 143-150, 1997.

[40] W. Cui, S. Sun, J.-a. Fang, Y. Xu, and L. Zhao, "Finite-time synchronization of Markovian jump complex networks with partially unknown transition rates," Journal of the Franklin Institute, vol. 351, no. 5, pp. 2543-2561, 2014. 